DOI: 10.4081/aiua.2021.1.86

\title{
Red man syndrome caused by intracavernous irrigation with vancomycin at the time of placing penile implants
}

Submitted 8 December 2020; Accepted 20 December 2020

To the Editor,

Erectile dysfunction is a condition that affects more than half of men between 40 and 70 years of age. Penile prosthesis (PP) implant is recognized, at present, as the most effective option to obtain an artificial erection satisfactory for sexual intercourse in those patients in which the pharmacological approach is contraindicated or ineffective (1). Postoperative infection is the most feared complication of genitourinary prosthetic surgery.

The literature contains several interventions that have been shown to reduce the rate of PP infection. One of the intraoperative approaches is to use an antiseptic washout solution containing vancomycin and gentamycin (2). We report a case of a 65-year-old male patient who developed red man syndrome (RMS) soon after the initiation of intracavernous irrigation of vancomycin during the implantation of a PP.

The case was successfully managed with diphenhydramine.

RMS is an idiopathic pseudo-allergic drug reaction that may develop after the administration of vancomycin. It is frequently observed with the infusion of vancomycin. The patient shows signs and symptoms of an allergic reaction, but without any classic allergy immunologic mechanism (3). Discontinuation of the vancomycin infusion and administration of diphenhydramine can abort most of the reactions. Slow intravenous administration of vancomycin should minimize the risk of infusion-related adverse effects (4).

A 65-year-old male with poorly controlled diabetes mellitus type 2 presented for inflatable PP insertion. He has no known food or drug allergies. On pre-procedure assessment, his blood pressure was 110/80 $\mathrm{mmHg}$, heart rate 72 beats per minute, respiratory rate 13 per minute, and temperature $37.1 \mathrm{C}^{\circ}$. In the supine position, prepping and draping in the usual sterile manner done. Under spinal anesthesia, a penoscrotal incision was made. The dartos fascia is now exposed and incised. Skin hooks are placed.

The tunica albuginea of both corporas is exposed and the urethral catheter is palpated in the midline. A stab wound is made with a scalpel into each corpora and, using Metzenbaum scissors, a 3-cm vertical corporotomy is performed between two 2-0 stay sutures. Corporal dilatation is performed. Vigorous intracavernous washout was done using an antimicrobial solution (500 mg vancomycin and $80 \mathrm{mg}$ gentamicin sulfate diluted in a 1 liter of normal saline solution). After 5 minutes, erythema developed along the patient's right forearm. At the same time, the patient developed redness of his right neck and itching of his scalp, mainly on his left side.

Adequate oxygenation by a face mask was initiated. Vital signs were stable. This was identified as RMS secondary to vancomycin.

The adverse reaction was resolved after the discontinuation of intracavernous irrigation with vancomycin and administration of diphenhydramine. The planned procedure was completed.

This report of RMS after intracavernous irrigation with vancomycin is believed the first in the literature.

The patient's symptoms disappeared within one hour after the medication was discontinued.

This case demonstrates the need to closely monitor for side effects of intracavernous irrigation with vancomycin and to carefully consider this approach.

\section{REFERENCES}

1. Bettocchi C, Palumbo F, Spilotros M, et al. Penile prostheses. Ther Adv Urol. 2010; 2:35-40.

2. Swanton AR, Munarriz RM, Gross MS. Updates in penile prosthesis infections. Asian J Androl. 2020; 22:28-33. 
3. Arroyo-Mercado F, Khudyakov A, Chawla GS, et al. Red man syndrome with oral vancomycin: a case report. Am J Med Case Rep. 2019; 7:16-17.

4. Sivagnanam S, Deleu D. Red man syndrome. Crit Care. 2003; 7:119-20.

\begin{abstract}
Mohamad Moussa ${ }^{1}$, Mohamad Abou Chakra ${ }^{2}$, Athanasios Papatsoris ${ }^{3}$, Athanasios Dellis ${ }^{4}$, Yasmine Moussa ${ }^{5}$

${ }^{1}$ Urology Department, Zahraa Hospital, University Medical Center, Lebanese University, Beirut, Lebanon;

${ }^{2}$ Faculty of Medicine, Department of Urology, Lebanese University, Beirut, Lebanon

${ }^{3} 2^{\text {nd }}$ Department of Urology, School of Medicine, Sismanoglio Hospital, National and Kapodistrian University of Athens, Athens, Greece;

${ }^{4}$ Department of Urology/General Surgery, Areteion Hospital, Athens, Greece;

${ }^{5}$ Clinic of Dermatology, Dr. Brinkmann, Schult \& Samimi-Fard, Gladbeck, Germany.
\end{abstract}

\title{
Correspondence
}

Mohamad Moussa, MD

mohamadamoussa@hotmail.com

Head of Urology Department, Zahraa Hospital, University Medical Center,

Lebanese University, Beirut, Lebanon

Mohamad Abou Chakra, MD (Corresponding Author)

mohamedabouchakra@hotmail.com

Faculty of Medicine, Department of Urology, Lebanese University, Beirut, Lebanon, 1108

Athanasios Papatsoris, MD

agpapatsoris@yahoo.gr

$2^{\text {nd }}$ Department of Urology, School of Medicine, Sismanoglio Hospital,

National and Kapodistrian University of Athens, Athens, Greece.

Athanasios Dellis, MD

aedellis@gmail.com

Department of Urology/General Surgery, Areteion Hospital, Athens, Greece

Yasmine Moussa, MD

moussa.yasmin@yahoo.com

Clinic of Dermatology, Dr. Brinkmann, Schult \& Samimi-Fard

Barbarastraße 15, 45964 Gladbeck, Germany 